\title{
NEAR-FIELD MEASUREMENT OF A PLANAR META-SURFACE ILLUMINATED BY DIPOLE ANTENNAS
}

\author{
Elena Saenz ${ }^{(1)}$, Kaan Guven ${ }^{(2)}$, Iñigo Ederra ${ }^{(1)}$, \\ Ekmel Ozbay ${ }^{(2)}$, Peter de Maagt ${ }^{(3)}$ and Ramon Gonzalo ${ }^{(1)}$ \\ (1) Electrical and Electronic Engineering Department, Public University of Navarra, \\ Campus Arrosadia, E-31006, Pamplona, Navarra, Spain \\ Email: elena.saenz@unavarra.es, ramon@unavarra.es, inigo.edera@unavarra.es
}

(2) Nanotechnology Research Center - NANOTAM, Department of Physics, Department of Electrical and Electronics Engineering Bilkent University, 06800, Ankara, Turkey

Email: guven@fen.bilkent.edu.tr,ozbay@bilkent.edu.tr

(3) European Space Research and Technology Centre, ESTEC, P.O. Box 299, 2201 AG Noordwijk, The Netherlands

Email: Peter.de.Maagt@esa.int

\begin{abstract}
In this paper, the uniform illumination of a meta-surface that is fed by a single dipole antenna or an array is experimentally investigated by means of near-field measurements. The results of the scanned field, when the dipoles are radiating in free space and when the meta-surface is placed atop them are presented. By means of this measurement, the coupling reduction between dipoles of an array due to the presence of the meta-surface is observed.
\end{abstract}

\section{Introduction}

One of the proposed applications of metamaterials is their use as superstrate for planar antennas in order to enhance their radiation performance. Several solutions have been proposed for this purpose. As an example, Partially Reflective Surfaces (PRS) in combination with a ground plane have been used to create a Fabry Perot like cavity [1]. By properly selecting the distance between the ground plane and the superstrate (around $\lambda / 2$ with a regular Perfectly Electric Conductor or $\lambda / 4$ if an Artificial Magnetic Conductor is used) as well as working at the boundary between the transmission pass and stop bands, a gain enhancement could be achieved. The solution proposed herein is conceptually different, in which no cavity and ground plane are required. The superstrate is placed in close proximity to the radiating source, and when working at the transmission pass band, the in-phase oscillation of the meta-surface's resonant inclusions is exploited [2]- [3].

The meta-surface that was used in this work is schematically shown in Fig. 1 (a) and it has been described in detail in [2]- [3]. It consists of two grids of parallel short dipoles and a grid of continuous wires in between. The heuristical operation can be explained in the following manner. Under normal incident plane-wave excitation, at the resonant frequency, out-of-phase currents are induced in the pairing dipoles that are seated in layers 1 and 3 . Since the distance $h$ between layers is very small compared to the wavelength, a closed loop is formed that creates a strong magnetic dipole moment that produces radiation, i.e. a transmission window appears (see Fig. 1 (c)). To widen the passband, a grid of solid wires is placed in between the dipole grids. Owing to the in-phase oscillation of the meta-surface inclusions, uniform illumination is obtained.

In this work, the meta-surface is illuminated by a single dipole or a $2 \times 2$ dipole antenna array. By means of near-field (NF) measurements of the radiated field, the surface illumination and the coupling between dipoles in the case of the array is investigated. 


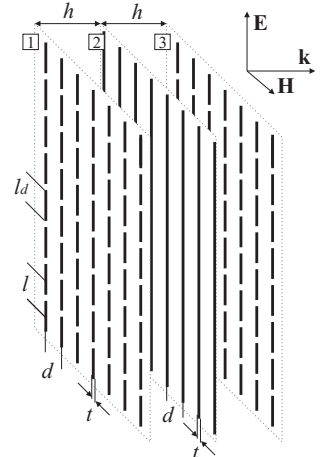

a)
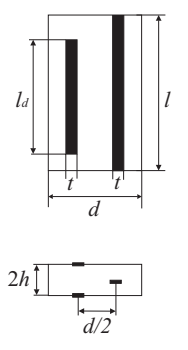

b)

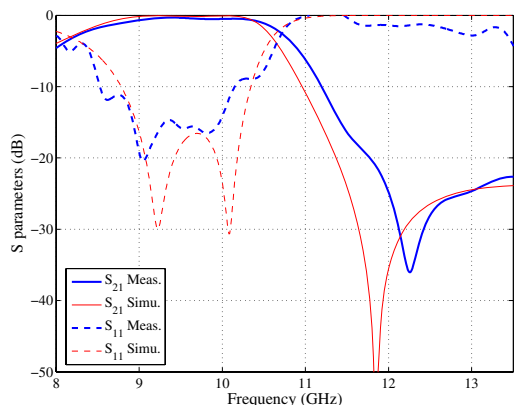

Figure 1: (a) A schematic representation of the proposed meta-surface. (b) Unit cell geometry: top and lateral views. (c) Simulated and measured transmission and reflection coefficients.

\section{Near-field measurements}

The field radiated by the antenna was tested by using a NF scanner and compared to the one radiated in the presence of the meta-surface. The set-up for the measurements is shown in Fig.2. The monopole antenna used as a probe was mounted over a $2 \mathrm{D}$ scanning system and placed $\lambda / 5$ far away from the antenna. The distance from the antenna to the probe $z_{0}$ was chosen so that the probe was close enough to measure the surface illumination but out of the reactive near field of the antenna, which is usually taken as $\lambda / 2 \pi$ [4]. Measurements at larger distances were also carried out, obtaining similar results after the back projection to the aperture. Since the measurement distance $z_{0}$ is smaller than $\lambda$, the sample points $\Delta_{x}$ and $\Delta_{y}$ should be chosen to be smaller than $\lambda / 2$ in order to satisfy the sampling criteria [4]- [6].

$$
\Delta_{x}, \Delta_{y}=\frac{\lambda}{2 \sqrt{1+\left(\frac{\lambda}{z_{0}}\right)^{2}}}
$$

Applying (1), for a working frequency of $10.5 \mathrm{GHz}$ and $z_{0}=\lambda / 5$, the sampling step should be around $\lambda / 10$, and therefore $\Delta_{x}$ and $\Delta_{y}$ were fixed to $3 \mathrm{~mm}$. The $\mathrm{E}$ field radiated by the antenna is shown to be along the metal strips, so, taking into account the radiation pattern of the monopole, this was oriented parallel to the surface. Details of the probe orientation regarding the antenna are shown in Fig. 3. In order to feed the antenna and measure the field that was to be detected by the probe, a vector network analyzer HP8510C was connected via GPIB to a PC was used.

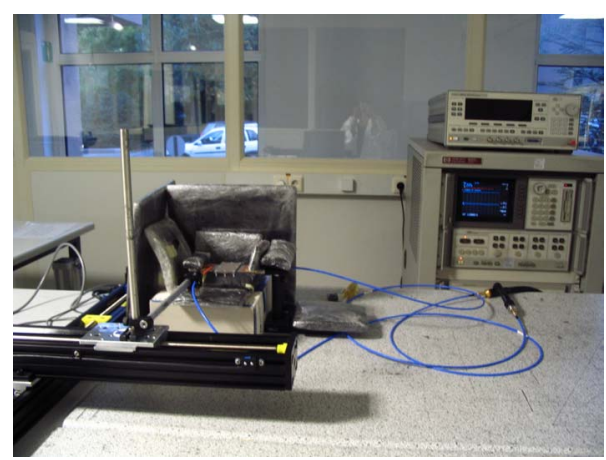

Figure 2: Set-up for the near-field measurements: vector network analyzer, 2D scanning system, probe, and antenna.

\section{Dipole antenna}

A dipole antenna was designed to be used as feeding element of the meta-surface. A picture of the dipole with and without the superstrate is shown in Fig. 3. The size of the superstrate is $13.6 \times 29.51 \mathrm{~mm}$ 
(approx. $0.47 \lambda \times 1 \lambda$ at the resonant frequency of $10.5 \mathrm{GHz}$ ). The distance between the dipole and the superstrate is $1.574 \mathrm{~mm}$, which has been adjusted in order to have good impedance matching. The overall thickness of the antenna (printed dipole plus superstrate) is $0.137 \lambda$.

By using the described NF set-up, the field radiated by the dipole was scanned over a $3 \lambda \times 3 \lambda$ surface. The results of the scanned field, when the dipole was radiating at the resonant frequency in free space and in the presence of the meta-surface, are shown in Fig. 3 (a) and (b), respectively. Due to the loading effect of the meta-surface, a frequency shift between the resonant frequency of the antenna radiating in free space $\left(f_{r}=9.51 \mathrm{GHz}\right)$ and the antenna with superstrate $\left(f_{r}=10.5 \mathrm{GHz}\right)$ is produced. In the absence of superstrate (Fig. 3 (a)) the regular radiation of a dipole radiating in free space is observed, i.e., broad beam in the H-plane and nulls in the endfire direction of the E-plane. However, when the meta-surface is placed on top of the dipole (Fig. 3 (a)), the radiated field is confined by the superstrate and radiated in the boresight radiation producing the gain enhancement. Moreover, a reduction of the endfire radiation is obtained all around the antenna. Measurements of the input impedance matching $\left(S_{11}=-15 \mathrm{~dB}\right.$ at 10.5 $\mathrm{GHz})$ and gain enhancement $(\mathrm{G}=6.8 \mathrm{dBi}$ at $10.5 \mathrm{GHz})$ in the presence of the superstrate are shown in Fig. $3(\mathrm{c})$.

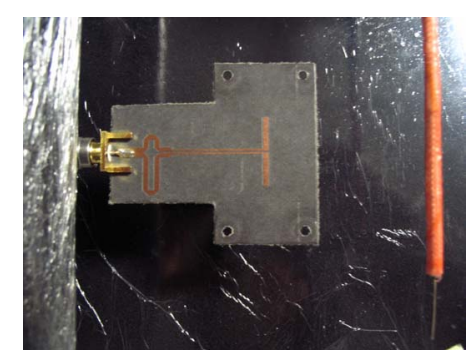

$9.51 \mathrm{GHz}$

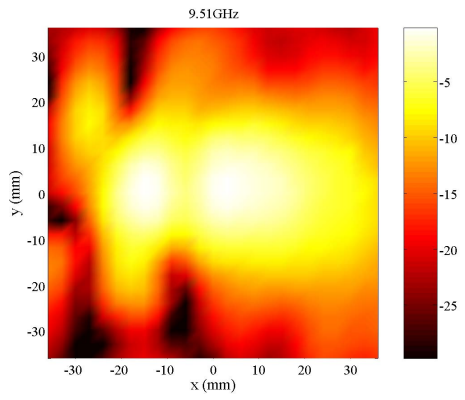

(a)

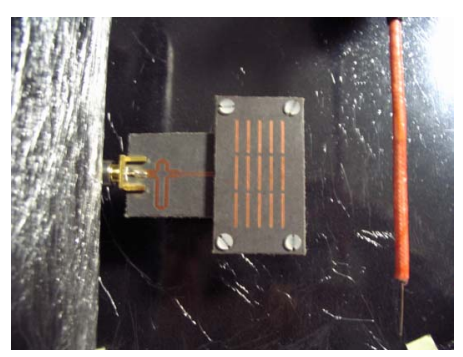

$10.5 \mathrm{GHz}$

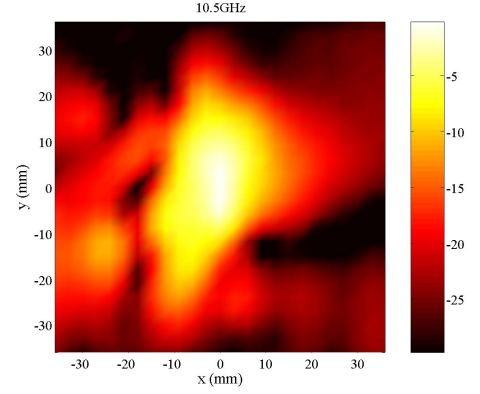

(b)

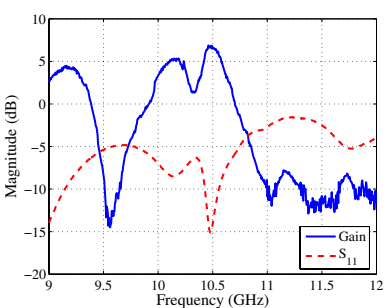

(c)

Figure 3: Antenna configurations and scanned field at the resonant frequency of each configuration. (a) Dipole radiating in free space; $f_{r}=9.51 \mathrm{GHz}$. (b) Dipole plus 5-periods superstrate; $f_{r}=10.5 \mathrm{GHz}$. (c) Input impedance matching and gain.

\section{Dipole antenna array}

The mutual coupling of a $2 \times 2$ dipole antenna array with a H-plane distance $0.5 \lambda$ and flipped upside down, i.e., baluns on the bottom face, was also investigated by NF measurements (see Fig. 4). One of the dipoles was fed and the others matched with $50 \Omega$ loads. By this measurement, the surface illumination and the field that reaches the neighbour dipoles can be detected. The probe was placed $\lambda / 5$ far away from the antenna and the step of the acquisition points $\Delta_{x}, \Delta_{y}$ was fixed to $2 \mathrm{~mm}$ so that the sampling criteria (1) was satisfied.

The results of the scanned field when the dipole placed at the top-left corner was radiating in free space and in the presence of the meta-surface are shown in Fig. 4 (a) and (b) respectively. The measure was done at the corresponding resonant frequency of each antenna $(9.3 \mathrm{GHz}$ in free space and $10.55 \mathrm{GHz}$ with the superstrate). It can be observed that, when the dipole is radiating in free space (Fig. 4 (a)), the main coupling occurs in the H-plane direction, being the E-plane and cross coupling quite low. However, in the presence of the superstrate (Fig. 4 (b)), the power radiated by the antenna is confined in the surface just over the active dipole and radiated in the boresight direction reducing the endfire radiation. Clearly 


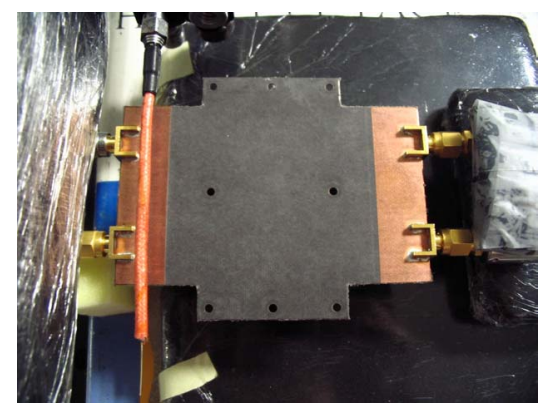

$9.26 \mathrm{GHz}$

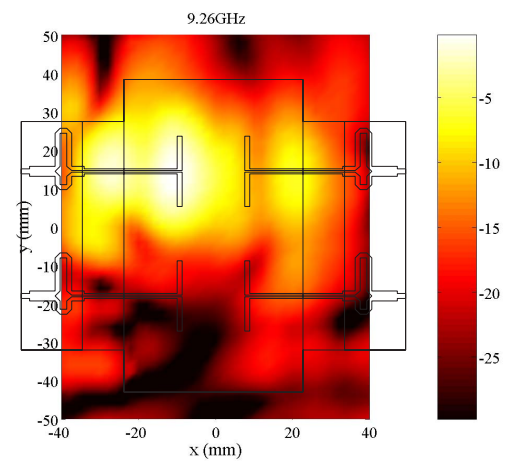

(a)

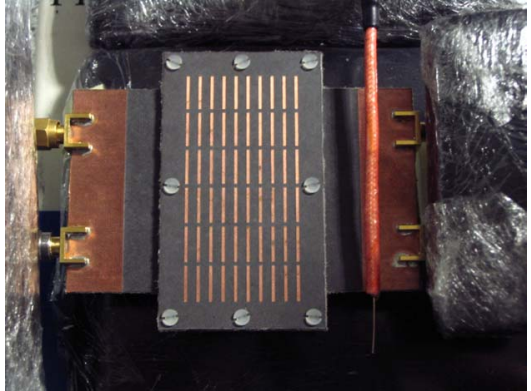

$10.55 \mathrm{GHz}$

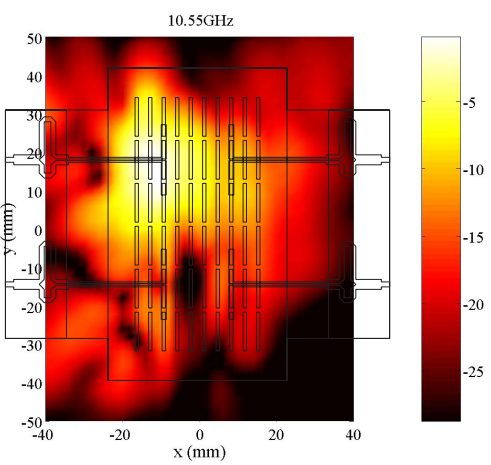

(b)

Figure 4: Near-field measurements of the dipole antenna array when dipole 1 (top, left) is radiating at the resonant frequency. Notice that the array is flipped upside down (baluns on the bottom face). (a) Printed dipoles without superstrate. (b) Dipoles with superstrate.

a coupling reduction in the H-plane is observed with similar levels in the E-plane and cross couplings. These results are consistent with the ones obtained by the $S$-parameters measurements (see [7]).

\section{Conclusions}

In this paper, the gain enhancement of a dipole antenna with a planar meta-surface as superstrate due to the uniform illumination of the meta-surface has been experimentally demonstrated. Near-field measurements of the E-field distribution on the radiating surface have proven the confinement of the power radiated by the dipole antenna, reducing the endfire radiation and improving the boresight radiation. In the case of an array, due to this enhancement of the radiation characteristics, a coupling reduction between elements mainly in the H-plane has been observed.

\section{References}

[1] Feresidis, A. P., and Vardaxoglou, J. C.: 'High gain planar antenna using optimized partially reflective surfaces', IEE Proc. Microwaves, Antennas and Propag., vol. 148, no. 6, 2001.

[2] Ikonen, P. M. T., Saenz, E., Gonzalo, R., and Tretyakov, S. A.: 'Modeling and Analysis of Composite Antenna Superstrates Consisting on Grids of Loaded Wires', IEEE Antennas and Propagation, vol. 55, no. 10, 2007.

[3] Saenz, E., Ederra, I., de Maagt, P., and Gonzalo, R.: 'High efficient dipole antenna with a planar metasurface', Elect. Lett., vol. 43, no. 16, 2007.

[4] Yaghjian, A.D.: 'An overview of near-field antenna measurements', IEEE Trans. Antennas Propag., vol. 34, no. 1, pp. 20-45, 1986.

[5] Looand, Y.T., and Lee, S.W. (Eds.): 'Antenna Handbook' Ch. 33 Near-Field Far-Field Antenna Measurements, J. Appel-Hansen, Van NostrandReinhold Company, NY, 1988.

[6] Rudge, A.X., Milne, K., Olver, A.D., and Knight, P. (Eds.): 'The Handbook of Antenna Design' Ch. 8, Peter Peregrinus Ltd., London, UK, 1982.

[7] Gonzalo, R., Saenz, E., Ederra, I., Ikonen, P. Tretyakov, S., and de Maagt, P.: 'Meta-surfaces for antenna applications', Proceedings EuCAP $200 \%$. 